\title{
Squeezed states of light from an optical parametric oscillator
}

\author{
Ling-An Wu, Min Xiao, and H. J. Kimble \\ Department of Physics, University of Texas at Austin, Austin, Texas 78712
}

Received May 12, 1987; accepted July 7, 1987

\begin{abstract}
Squeezed states of the electromagnetic field are generated by degenerate parametric downconversion in a subthreshold optical parametric oscillator. Reductions in photocurrent noise greater than $60 \%(-4 \mathrm{~dB})$ below the limit set by the vacuum fluctuations of the field are observed in a balanced homodyne detector. A quantitative comparison with theory suggests that the observed noise reductions result from a field that in the absence of avoidable linear attenuation would be squeezed more than tenfold. A degree of squeezing of approximately fivefold is inferred for the actual field emitted through one mirror of the optical parametric oscillator. An explicit demonstration of the Heisenberg uncertainty principle for the electromagnetic field is made from the measurements, which show that the field state produced by the downconversion process is a state of minimum uncertainty.
\end{abstract}

\section{INTRODUCTION}

The quantum-statistical properties of the fields generated by optical parametric amplification have been investigated since the earliest days of quantum optics more than 25 years ago. ${ }^{1-4}$ Of particular interest has been the nonclassical nature of the fields produced by parametric downconversion from an intense pump beam into signal and idler modes that are intially in the vacuum state. ${ }^{5-7}$ A quantum treatment of this process is of course essential since the unexcited modes gain excitation only by way of spontaneous parametric fluorescence, in which the high-frequency pump photons are split into highly correlated pairs of lower-frequency signal and idler photons. From the perspective of squeezed-state generation, it is precisely the high degree of nonclassical correlation between the downconverted photons that gives the field state its unique properties. In the simplest possible case of a nondepleted degenerate parametric amplifier with plane-wave inputs, the time evolution of the subharmonic field is described by a Bogoliubov transformation that maps the initial vacuum state into a squeezed state of minimum uncertainty. ${ }^{8-10}$ This transformation arises from a Hamiltonian quadratic in photon annihilation and creation operators (photons removed and inserted in pairs in the subharmonic mode) and is equivalent to a description in terms of canonically conjugate quadrature-phase operators for which the phase space is stretched along one dimension and contracted along an orthogonal dimension. ${ }^{11}$ For an initial vacuum state, this elastic deformation of the phase space produces a squeezed vacuum with an asymmetric distribution of fluctuations. ${ }^{12}$

A possible experimental realization of squeezed-state generation by parametric downconversion would be a singlepass amplifier for which squeezing over a bandwidth comparable with the phase-matching bandwidth could be realized. While such an experiment might well be feasible with new generations of nonlinear optical materials ${ }^{13}$ or with pulsed input trains, ${ }^{14}$ our effort has centered instead on an investigation of parametric downconversion inside an optical cavity. The resulting configuration is termed an optical parametric oscillator (OPO), for which there exists an extensive literature. ${ }^{15,16}$ If the signal and idler modes of the oscillator are both resonant in the cavity, the OPO is called doubly resonant. The arrangement that we consider is a triply resonant cavity with the pump mode and the nearly degenerate signal and idler fields simultaneously resonant. We concentrate on the below-threshold operation of this device. Yurke ${ }^{17}$ first showed that parametric amplification and deamplification in this regime could, in fact, lead to arbitrarily large degrees of squeezing in an analysis that selfconsistently couples the traveling-wave, single-pass characteristics of the downconversion process with the boundary conditions imposed in a single-sided optical cavity. Collett and Gardiner ${ }^{18}$ and Gardiner and Savage ${ }^{19}$ then presented wideband analyses to predict the actual spectral distribution of squeezing of the field emitted by the OPO below threshold.

While these theories are directly applicable to the case of a single-mode OPO (one pump mode, one signal mode, one idler mode), we demonstrate that they are as well applicable to a subthreshold multimode OPO (one pump mode, but a multitude of pairs of cavity modes for signal and idler fields that satisfy both the cavity resonance conditions and the phase-matching requirements). This is an extremely important recognition since it permits one to construct and operate a low-loss, single-port cavity without incorporating frequency-selective elements to enforce degenerate operation and without the technical difficulties that accompany stable operation above threshold.

The experiments that we describe in this paper investigate squeezed-state generation in such a multimode OPO for frequencies close to the point of degeneracy. We report observed noise reductions in a homodyne detector of greater than $60 \%$ relative to the level set by the vacuum state of the field. From the recorded noise reductions together with separate, absolute measurements of propagation and detection efficiencies, we are able to infer that the field produced by the subthreshold OPO is in fact almost ideally squeezed (at a level of 10 times squeezing). By considering both enhancements and reductions in the fluctuations of the field, we further infer that the field is generated in a minimum-uncertainty state. Degradation of this state appears to occur by a series of avoidable linear-loss mechanisms present in the current apparatus. Within the current ex- 
perimental uncertainties, nothing fundamental in the parametric process itself or in the materials employed was found that intrinsically limits the achievable squeezing. In the context of other recent reports of squeezed-state generation, ${ }^{20-25}$ our results for the OPO represent by far the largest degrees of observed noise reduction and inferred level of squeezing.

The paper is organized as follows: In Section 2 we recall relevant results from the theoretical literature on squeezing with the OPO. We give an explicit expression for the coupling coefficient $\kappa$ that allows us to estimate the power required to reach the threshold for parametric oscillation and hence to produce significant degrees of squeezing. Section 3 is a discussion of the principal elements of the experiment. In Section 4 we consider in detail the operation of the balanced homodyne receiver for detecting squeezed light. Our observations of noise reductions below the vacuum level and the inferred degrees of squeezing of the field are described in Section 5 . Section 6 serves as a conclusion.

\section{THEORETICAL OVERVIEW}

The configuration of the OPO to be considered is illustrated in Fig. 1. A nonlinear crystal characterized by second-order susceptibility $\chi^{(2)}$ is contained in the cavity formed by the mirrors $\left(M, M^{\prime}\right)$, which are highly reflective at both fundamental and subharmonic frequencies. Excitation is provided by coherent fields of amplitudes $\left(E_{1}, E_{2}\right)$ and frequencies $(\omega, 2 \omega)$, respectively. The semiclassical equations of motion for this system are readily obtained from Maxwell's equations driven by the nonlinear polarization as the source term. We follow the treatment of Drummond et al. ${ }^{26}$ and references therein to write

$$
\begin{aligned}
& \dot{\alpha}_{1}=-i \Delta_{1} \alpha_{1}-\Gamma_{1} \alpha_{1}+\kappa \alpha_{1}{ }^{*} \alpha_{2}+E_{1}, \\
& \dot{\alpha}_{2}=-i \Delta_{2} \alpha_{2}-\Gamma_{2} \alpha_{2}-1 / 2 \kappa \alpha_{1}^{2}+E_{2} .
\end{aligned}
$$

The intracavity amplitudes $\alpha_{i}$ at the subharmonic $(i=1)$ and fundamental $(i=2)$ frequencies are normalized such that $\left|\alpha_{i}\right|^{2}$ expresses the mean photon number in the cavity mode $i$. $\alpha_{1}$ and $\alpha_{2}$ are slowly varying amplitudes defined in rotating frames of frequencies $\omega$ and $2 \omega$. The detunings $\Delta_{i}$ from the cavity resonances at $\omega_{i}$ are given by $\Delta_{1}=\omega_{1}-\omega$ and $\Delta_{2}=\omega_{2}-$ $2 \omega$, while the mode amplitudes are damped at rates $\Gamma_{i}$. The coupling coefficient $\kappa$ is proportional to $\chi^{(2)}$ and is given in explicit form below.

By examining the steady-state solutions $\alpha_{i}^{0}$ for the case $\Delta_{1}$ $=0=\Delta_{2}$ and $E_{1}=0$, one finds from Eqs. (1) that the threshold value of $E_{2}$ for parametric oscillation is $E_{2} \mathrm{c}=\Gamma_{1} \Gamma_{2} /$ $\kappa$. Below this value $\alpha_{1}{ }^{0}=0$ and $\alpha_{2}{ }^{0}=E_{2} \Gamma_{2}$, while for $E_{2}>$ $E_{2}{ }^{c}$, the stable solutions are

$$
\left|\alpha_{1}^{0}\right|=\left[\frac{2}{\kappa}\left(E_{2}-E_{2}^{c}\right)\right]^{1 / 2}, \quad\left|\alpha_{2}^{0}\right|=\Gamma_{1} / \kappa .
$$

A linear stability analysis about these steady states reveals that the instability at threshold is associated with a loss of stability for the modulus $A_{1}$ of the complex field $\alpha_{1}$, where $\alpha_{1}$ $=A_{i} e^{i \beta_{i}}$. As the threshold for parametric oscillation is approached, the effective damping coefficient for amplitude deviations of the subharmonic approaches zero. Phase deviations, on the other hand, are still strongly damped. This observation leads one to surmise that, if squeezed states are

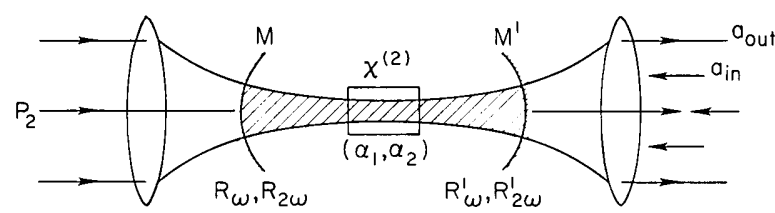

Fig. 1. Sketch of basic arrangement of the optical parametric oscillator. The pump beam $P_{2}$ at frequency $2 \omega$ excites the cavity through the mirror $M$. The downconverted field of approximate frequency $\omega$ decays predominantly through the mirror $M^{\prime}$. The system can be used as an amplifier by injecting a coherent field $a_{\text {in }}$ at the subharmonic frequency through $\mathrm{M}^{\prime}$.

generated in this system, they will be produced with reduced phase and enhanced amplitude fluctuations.

To pursue somewhat further this qualitative discussion of the classical basis for squeezed-state generation, we consider the subthreshold $\left(E_{2}<E_{2}{ }^{c}\right)$ OPO as an amplifier. ${ }^{18,26}$ For the case of $\mathbf{M}^{\prime}$ as the input coupler for an additional weak coherent probe field of amplitude $a_{\text {in }}$ and frequency $\omega_{\text {in }}=\omega$, we calculate $G \equiv a_{\text {out }} / a_{\text {in }}$ for the ratio of the amplitude $\dot{a}_{\text {out }}$ of the total emitted field to the amplitude $a_{\text {in }}$ of the incident field. We assume that both modes are resonantly excited $\Delta_{1}$ $=0=\Delta_{2}$ and that cavity losses other than through the transmission of $\mathrm{M}^{\prime}$ are zero. Of course, we must, in addition, specify the phase $\phi$ of the probe field relative to that of the pump field $E_{2}$, which we take to be real and positive. For special cases $\phi=0$ and $\phi=\pi / 2$ with corresponding gains $G_{+}$ and $G_{-}$, respectively, we find by applying the appropriate boundary conditions at $\mathrm{M}^{\prime}$ that

$$
\begin{aligned}
& G_{+}=\frac{1+d}{1-d}, \\
& G_{-}=\frac{1-d}{1+d},
\end{aligned}
$$

where we have assumed that $A_{1} \ll 1$ and defined $\dot{d} \equiv E_{2} / E_{2}{ }^{c}$. A field of classical amplitude $a_{\text {in }}$ incident upon this cavity will thus experience gain $G_{+}$or loss $G_{-}$, depending on its phase $\phi$ relative to that of the pumping field $E_{2}$. A similar statement holds for coherent AM or FM modulation or indeed for any classical noise that may accompany $a_{\text {in }}$, so long as the frequency of modulation is small compared with $\Gamma_{1}{ }^{27}$ We therefore find in this simple analysis all the ingredients necessary for squeezed-state generation, including the possibility of producing minimum-uncertainty states since $G_{+} G_{-}$ $=1$ for all $d<1$.

A quantitative description of the nature of the quantum fluctuations in this system can in fact be obtained precisely along the lines outlined above, but with Hilbert-space operators replacing the classical fields. ${ }^{17-19}$ As before, we work in a rotating frame of frequency $\omega$ for the subharmonic field and introduce annihilation and creation operators $\left[\hat{\alpha}_{1}(t), \hat{\alpha}_{1}^{\dagger}\right.$ $(t)$ ] for the intracavity field, where the caret denotes a Hilbert-space operator. The quadrature amplitude $\hat{X}_{\theta}(t)$ of the intracavity field is defined by

$$
\hat{X}_{\theta}(t) \equiv e^{-i \theta} \hat{\alpha}(t)+e^{i \theta} \hat{\alpha}^{\dagger}(t),
$$

with associated amplitude correlation function

$$
A(\tau, \theta) \equiv\left\langle T: \hat{X}_{\theta}(t), \hat{X}_{\theta}(t+\tau):\right\rangle,
$$

where the colons denote normal ordering, $T$ stands for time ordering, the covariance $\langle\hat{a}, \hat{b}\rangle \equiv\langle\hat{a} \hat{b}\rangle-\langle\hat{a}\rangle\langle\hat{b}\rangle$, and the 
time $t$ is sufficiently long to ensure a (stationary) steady state. The spectrum of squeezing $S(\nu, \theta)$ of the output field from an ideal single-ended cavity of damping rate $\Gamma_{1}$ is then given in terms of the spectral density $A(\nu, \theta)$ by

$$
S(\nu, \theta)=2 \Gamma_{1} A(\nu, \theta),
$$

where

$$
A(\nu, \theta)=\int_{-\infty}^{\infty} \mathrm{d} \tau e^{-i \nu \tau} A(\tau, \theta)
$$

That is, the intracavity fluctuations described by $A(\nu, \theta)$ decay through the output coupler $\mathbf{M}^{\prime}$ into the continuum of external modes propagating away from the cavity to give a spectrum of squeezing $S(\nu, \theta)$. The critical steps in this derivation are found in the work of Ref. 18. Note that any external fields incident upon the cavity at the subharmonic are assumed to be in either a vacuum or a coherent state. Since $S(\nu, \theta)$ is a normally ordered quantity, $S(\nu, \theta)=0$ for the vacuum state, whereas from the definition of Eq. (3), $S(\nu$, $\left.\theta_{0}\right) \equiv S_{-}(\nu) \rightarrow-1$ and $S\left(\nu, \theta_{0}+\pi / 2\right) \equiv \mathrm{S}_{+}(\nu) \rightarrow \infty$ for perfect squeezing, with $\theta_{0}$ chosen for optimum squeezing in one quadrature-phase amplitude.

As for the squeezing produced by the OPO, a single-mode analysis by Yurke ${ }^{17}$ showed that arbitrarily large degrees of squeezing should be obtainable in the output field of a degenerate optical parametric oscillator around the point of threshold. An explicit expression for $S(\nu, \theta)$ was first given by Collett and Gardiner ${ }^{18}$ and by Gardiner and Savage. ${ }^{19} \mathrm{~A}$ more general expression including possible excitation $E_{1} \neq 0$ and operation both below and above threshold is due to Collett and Walls ${ }^{28}$ and is as follows:

$$
S_{ \pm}(\nu)=\frac{ \pm 4 \Gamma_{1}\left|\epsilon_{2}\right|\left(\Gamma_{2}^{2}+\nu^{2}\right)}{\left[\Gamma_{2}\left(\Gamma_{1} \mp\left|\epsilon_{2}\right|\right)+\left|\epsilon_{1}\right|^{2}-\nu^{2}\right]^{2}+\nu^{2}\left(\Gamma_{1} \mp\left|\epsilon_{2}\right|+\Gamma_{2}\right)^{2}},
$$

where $\epsilon_{1}=\kappa \alpha_{i}^{0}$ and $\Delta_{1}=0=\Delta_{2}$. For operation below threshold with $E_{1}=0$ (which is the case of interest in our current experiments), this equation reduces to

$$
S_{ \pm}(\Omega)= \pm \frac{4 E_{2} / E_{2}^{c}}{\Omega^{2}+\left(1 \mp E_{2} / E_{2}^{c}\right)^{2}},
$$

where we have made use of the relations $\epsilon_{2}=\kappa \alpha_{2}{ }^{0}=\kappa E_{2} / \Gamma_{2}$ and $\epsilon_{2}{ }^{c}=\Gamma_{1}$ and have introduced the dimensionless frequency $\Omega=\nu / \Gamma_{1}$. As anticipated in our classical analysis, amplitude fluctuations are enhanced $\left[S_{+}(\Omega)=S(\Omega, \theta=0)\right]$ for $E_{2} /$ $E_{2}{ }^{c} \equiv d \rightarrow 1$, while fluctations in phase $\left[S_{-}(\Omega)=S(\Omega, \theta=\pi /\right.$ $2)$ ] are strongly suppressed. Indeed, for $\Omega=0,\left(1+S_{ \pm}\right)=$ $G_{ \pm}{ }^{2}$, and perfect squeezing is approached for $d \rightarrow 1$, with the quantities $\left(1+S_{ \pm}\right.$) playing the roles of variances of the quadrature-phase amplitudes of the external field. ${ }^{29}$ For this problem as for many other examples in quantum optics, a useful intuitive picture of squeezed-state generation is one in which the deterministic, classical gain functions operate in a phase-sensitive fashion on the quantum fluctuations of the field to produce squeezing. Note that from Eq. (8), $(1+$ $\left.S_{+}\right)\left(1+S_{-}\right)=1$ for the subthreshold OPO, so that the field produced should be in a state of minimum uncertainty. ${ }^{8-10,29}$

As a conclusion to this theoretical overview and before turning to a description of the experiment, we return to Eqs. (1) to evaluate the coupling coefficient $\kappa$ somewhat more carefully. It is, of course, this coefficient together with the cavity losses that sets the scale for the power level at threshold and hence for the feasibility of the experiment. In general, $\kappa$ is expressed in terms of an overlap integral of the cavity mode functions $\Psi_{i}(\mathbf{r})(i=1,2)$ as

$$
\kappa=\frac{2 d_{e}}{n^{3}}\left(\frac{h \omega_{1}^{3}}{\epsilon_{0}^{3}}\right)^{1 / 2} \int_{\Gamma} \Psi_{2}^{*}(\mathbf{r})\left[\Psi_{1}(\mathbf{r})\right]^{2} \mathrm{~d}^{3} x,
$$

with $\Gamma$ as the interaction volume and $d_{e}$ the effective nonlinear coefficient. ${ }^{30}$ By substituting the TEM $_{\infty}$ mode functions suitable for a standing-wave interferometer filled with medium of index $n$, we find by neglecting double refraction (that is, we assume $90^{\circ}$ phase matching) and linear absorption that

$$
|\kappa|^{2}=\frac{\pi c^{4} \hbar}{L^{3} \lambda_{2} n^{3}} E_{\mathrm{NL}}
$$

Here $L=$ cavity length and $\lambda_{i}$ is the free-space wavelength at frequency $\omega_{i}$. The nonlinear conversion efficiency $E_{\mathrm{NL}}$ is given by

$$
\begin{aligned}
E_{\mathrm{NL}} & =\frac{16 \pi^{2} l d_{e}^{2}}{\epsilon_{0}{ }^{3} c n^{2} \lambda_{1}^{3}} g(\sigma, \zeta), \\
g(\sigma, \zeta) & =\frac{\pi^{2}}{\zeta}\left|\operatorname{Im}\left\{H(\sigma, \zeta) \exp \left[i\left(\phi_{2}-2 \phi_{1}\right)\right]\right\}\right|^{2},
\end{aligned}
$$

with $l=$ crystal length, $\zeta=l / 2 Z_{0}, Z_{0}=$ cavity confocal parameter, $\sigma=Z_{0}\left(2 k_{1}-k_{2}\right), k_{i}=2 \pi n / \lambda_{i}$. The phases $\phi_{i}$ are those of the cavity mode functions $\Psi_{i}$ within the crystal; the factor $\left(\phi_{2}-2 \phi_{1}\right)$ expresses the relationship of the standingwave patterns of the two modes. $H(\sigma, \zeta)$ is defined as in Eq. (2.16) of Ref. 31 as,

$$
H(\sigma, \zeta) \equiv \frac{1}{2 \pi} \int_{-\zeta}^{\zeta} \mathrm{d} x \frac{e^{i \sigma x}}{1+i x}
$$

In the plane-wave limit, $\zeta \rightarrow 0$ and

$$
g(\sigma, \zeta) \rightarrow \zeta\left(\frac{\sin \sigma \zeta}{\sigma \zeta}\right)^{2} \sin ^{2}\left(\phi_{2}-2 \phi_{1}\right)
$$

The first factor expresses the usual condition for phase matching in a single pass. The second factor produces a modulation of the coupling efficiency with relative phase of the standing waves within the crystal. It is most easily understood by examining the case of frequency doubling $\left(E_{1}\right.$ $\neq 0, E_{2}=0$ ) for which there is a $90^{\circ}$ phase shift between the polarization wave and field at $\omega_{2}$. Thus the mode functions must be shifted by $\phi_{2}-2 \phi_{1}=\pi / 2$ within the crystal and by $\phi_{2}-2 \phi_{1}=0$ at the planes of the cavity mirrors. ${ }^{32}$ In the general case $\zeta \neq 0$, we require for optimal coupling

$$
\phi_{2}-2 \phi_{1}= \pm(\pi / 2)+\xi
$$

with $\xi$ the phase of the complex function $H$. When this condition is satisfied, the function $g(\sigma, \zeta)$ reduces to $h(\sigma, \zeta)$, with $h(\sigma, \zeta)$ as defined and analyzed in Eq. (2.22) and Fig. 2 of Ref. 31 .

Combining Eq. (10) and Eq. (A7) of Ref. 26, we find that the threshold condition for oscillation $E_{2}{ }^{c}=\Gamma_{1} \Gamma_{2} / \kappa$ can be expressed in terms of a critical power $P_{2}{ }^{c}$ for the pumping beam shown in Fig. 1, 


$$
P_{2}^{c}=\frac{\pi^{2}}{4 F_{\omega_{1}}^{2} B_{\omega_{2}} E_{\mathrm{NL}}} .
$$

Here, $F_{\omega_{1}}$ is the cavity finesse at the subharmonic cavity mode $\omega_{1} ; B_{\omega_{2}}$ is the buildup factor associated with the injected power at the cavity mode $\omega_{2}$ (one-way circulating power divided by $P_{2}$ in the absence of coupling, $\kappa=0$ ); and $E_{\mathrm{NL}}$ is the nonlinear conversion efficiency from Eq. (11). If condition (15) is satisfied, $E_{\mathrm{NL}}$ is operationally just the ratio $p_{2}{ }^{2} / p_{1}$ for frequency doubling, with power $p_{1}$ incident upon the crystal and power $p_{2}$ transmitted. In terms of the literature on optical parametric oscillators, the case we consider is that of a triply resonant cavity (pump, signal, and idler are all resonant). To set the stage for the next section, we estimate $P_{2}$ c for parameters typical of our experiment; that is, we take $F_{\omega_{1}} \sim 10^{2}, B_{\omega_{2}} \sim 10$, and $E_{\mathrm{NL}} \sim 3 \times 10^{-3} / \mathrm{W}$ and find $P_{2}{ }^{c} \sim 10$ $\mathrm{mW}$ for a cavity optimized with respect to Eq. (15). Note that Eq. (8) indicates that, for a pump power $P_{2}$ of only $30 \%$ of this threshold value, one should be able to achieve $S_{-}<$ -0.90 , corresponding to greater than 10 times squeezing.

\section{EXPERIMENTAL CONFIGURATION}

Our investigation of squeezed-state generation in the OPO concentrates on the below-threshold region. Although the analysis of the previous section indicates that the OPO should be an ideal candidate for producing large degrees of squeezing, the calculation is restricted to the case of a singlemode, degenerate OPO and does not deal with the other longitudinal modes of the cavity that are also phase matched and that participate in the downconversion process. Indeed, it is this multiplicity of modes symmetrically displaced around the point of degeneracy that leads to the well-known inherent instability of an OPO operated above threshold. ${ }^{15,16,30}$ The hopping of the oscillation frequency of the OPO over large intervals (characterized by the cluster spacing) is analogous to the mode hopping that occurs in a homogenously broadened laser operated above threshold and for which the longitudinal-mode spacing is small compared with the width of the gain curve. In the absence of intracavity frequency-selective elements, it is quite difficult to predict or to stabilize the frequency of oscillation of such a laser as one passes from below to above threshold. Note, however, that below threshold the inversion remains unsaturated and the individual modes live in a more or less egalitarian society in which the photon statistics of any given mode are more or less just those predicted by single-mode laser theory.

From a theoretical perspective, this analogy with the laser suggests that a multimode OPO should be well described by the theory of a single-mode OPO for operation below threshold. From an experimental viewpoint, a multimode cavity composed simply of two mirrors and a crystal is much more easily constructed and operated than is a single-mode cavity given the low gain per pass of the continuous-wave system that we envision. Although we have made preliminary investigations of single-mode, degenerate operation above threshold by employing an injected signal at the subharmonic to stabilize the system around degeneracy, we concentrate exclusively on the below-threshold regime in our discussion here.

A diagram of our experimental arrangement is shown in Fig. 2. The optical parametric oscillator consists of the

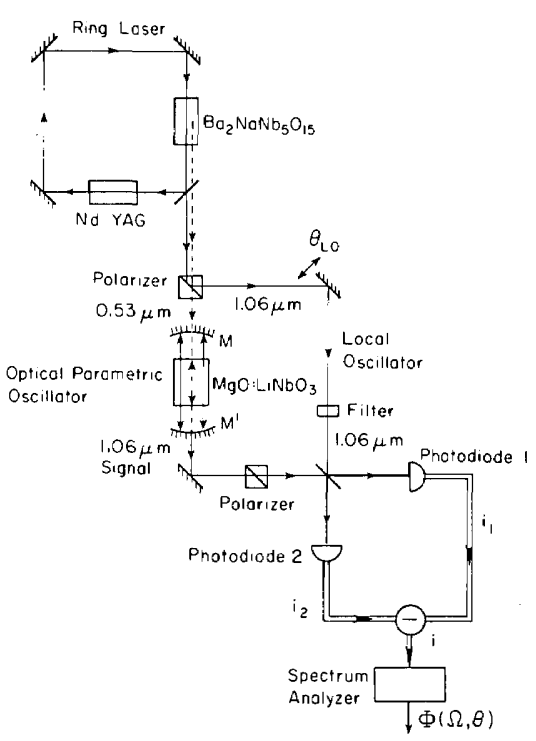

Fig. 2. Diagram of principal elements of the apparatus.

cavity $\left(\mathrm{M}, \mathrm{M}^{\prime}\right)$ containing a crystal of $\mathrm{MgO}: \mathrm{LiNbO}_{3}$ of dimension $25 \mathrm{~mm} \times 9 \mathrm{~mm} \times 8 \mathrm{~mm}$ heated to the phasematching temperature of $98^{\circ} \mathrm{C}$. Noncritical phase matching is employed to minimize effects from double refraction. The lithium niobate crystal is coated with dual-band antireflection coatings to minimize loss at 0.53 and $1.06 \mu \mathrm{m}$, resulting in transmissions of 96-97\% and 98-99\% at the respective wavelengths. The pump field at frequency $2 \omega$ enters the OPO cavity through the mirror $M$ that has transmission coefficients of $3.5 \%$ at $0.53 \mu \mathrm{m}$ and $0.06 \%$ at $1.06 \mu \mathrm{m} . \mathrm{M}^{\prime}$ is the output coupler for the subharmonic field at frequencies around $\omega$, with a transmission coefficient of either 4.3 or $7.3 \%$ (for the two cavities that we have studied) at $1.06 \mu \mathrm{m}$ and high reflectivity at $0.53 \mu \mathrm{m}$.

The pump $P_{2}$ at $0.53 \mu \mathrm{m}$ is generated with a crystal of $\mathrm{Ba}_{2} \mathrm{NaNb}_{5} \mathrm{O}_{15}$ inside the cavity of a Nd:YAG laser. As described in Ref. 33, the laser frequency is locked to the transmission peak of an external reference cavity to produce a rms linewidth of approximately $100 \mathrm{kHz}$. The orthogonally polarized components at frequencies $(\omega, 2 \omega)$ that emerge from the laser are separated by a polarizer. The green light at frequency $2 \omega$ is directed to the OPO cavity; the infrared emission at $1.06 \mu \mathrm{m}$ serves as a local-oscillator beam in a balanced homodyne detector. For most measurements, the intensities of both the local-oscillator beam and the intracavity green field are actively stabilized with servos based on acousto-optic modulators, with the first-order diffracted beam at $110 \mathrm{MHz}$ serving as a variable loss. [The degree of stability achieved for the local-oscillator power is shown in Fig. 4 below. The intracavity intensity at $0.53 \mu \mathrm{m}$ is held fixed to within \pm 0.03 . Without this stabilization, large $( \pm 0.20)$ variations of pump power produce variations in crystal temperature through linear absorption, sufficient to drive the system away from the condition of simultaneous resonance.] The length of the OPO cavity is servo controlled to lock a longitudinal-mode resonance to the frequency of the incident green beam at $0.53 \mu \mathrm{m}$ with an $\mathrm{rf}$ sideband technique employing phase modulation at 57 $\mathrm{MHz}^{34}$

A coarse search for the region of degenerate operation is 
initially conducted with the OPO cavity unlocked and with the length repetitively scanned through several spectral orders. A search in temperature then tunes the birefringence of the crystal to bring a longitudinal cavity mode at $1.06 \mu \mathrm{m}$ into simultaneous resonance with one at $0.53 \mu \mathrm{m}$. Over a rather broad range, this concidence of modes results in above-threshold oscillation for input powers $P_{2}>30 \mathrm{~mW}$ for the output coupler $\mathrm{M}^{\prime}$ with $R_{\omega} \sim 0.957$. Note that a plot of signal and idler frequencies versus crystal temperatures for a negative uniaxial crystal should be a parabolic function opening to the right along the abscissa (increasing temperature), with the nose of the parabola at the region of degenerate operation. We thus lower the temperature to identify the lowest temperature at which oscillation occurs. A filter of $10-\mathrm{nm}$ passband centered at $1.064 \mu \mathrm{m}$ facilitates this search. The definitive identification of degenerate oscillation is made by injecting an auxiliary beam from the laser at $\omega$ and observing the heterodyne signal with the OPO emission. The temperature window over which degenerate oscillation can be observed in this fashion is $\sim 0.3^{\circ} \mathrm{C}$, which is approximately equal to the observed FWHM of the phasematching curve separately obtained in extracavity frequency-doubling experiments $\left(\sim 0.3^{\circ} \mathrm{C}\right)$.

Given the observed thresholds for parametric oscillation, one can make a comparison with the prediction of Eq. (16). For these measurements, $F_{\omega_{1}} \sim 110, B_{\omega_{2}} \sim 8$, and $E_{\mathrm{NL}} \sim 7 \times$ $10^{-3} / \mathrm{W}$, leading to a calculated value of $P_{2} c \approx 4 \mathrm{~mW}$. The observed critical powers are in the range $10-15 \mathrm{~mW}$ for nondegenerate oscillation and $25-30 \mathrm{~mW}$ for degenerate oscillation. The discrepancy between observation and calculation is probably due to two sources. The first is that the cavity is operated close to the concentric limit so that slight variations in length from run to run can give rather large variations in the cavity waist and hence in $E_{\mathrm{NL}}$. Second, the rather stringent requirements on the relative phase of the mode functions imposed by the constraints of simultaneous resonance (for pump, signal, and idler) and appropriate overlap in the crystal ${ }^{32}$ are almost surely not optimally met in our cavity. We have performed preliminary experiments with xenon gas as a dispersive element in the spaces between the crystal and the mirrors. Variations of xenon pressure allow one to achieve both simultaneous resonance and opti-

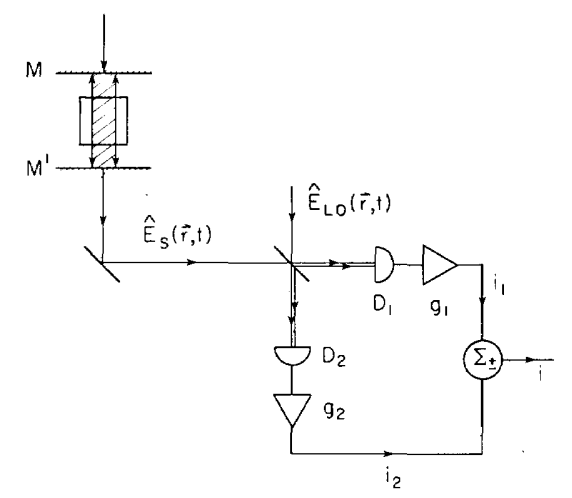

Fig. 3. Schematic of balanced homodyne detector. The signal field $\dot{E}_{S}$ from the optical parametric oscillator and a strong localoscillator field $\hat{E}_{\text {LO }}$ are combined at the surface of a beam splitter. The composite fields from the beam splitter are directed to photodiodes $\mathrm{D}_{1}$ and $\mathrm{D}_{2}$. The photocurrents are amplified with gains $\left(g_{1}, g_{2}\right)$ and summed in a hybrid junction with either a $0^{\circ}\left(\Sigma_{+}\right)$or a $180^{\circ}\left(\Sigma_{-}\right)$ phase shift. mum coupling, as defined in Eq. (15). The value of $E_{\mathrm{NL}} \sim 7$ $\times 10^{-3} / \mathrm{W}$ taken for the estimate of $P_{2}{ }^{c}$ is the maximum that one should expect for our crystal, in the optimum focusing geometry with Eq. (15) satisfied.

With the temperature range for degenerate oscillation identified as outlined above, the scan of cavity length is stopped, the incident power $P_{2}$ is reduced for operation below threshold, and the frequency servo is engaged to lock a longitudinal resonance at $0.53 \mu \mathrm{m}$ to the frequency of the incident green beam. The crystal temperature is then slowly varied to bring successive longitudinal modes at $1.06 \mu \mathrm{m}$ into simultaneous resonance with the strong intracavity field at $0.53 \mu \mathrm{m}$. A fraction of the downconverted light resulting from the interaction in the OPO exits through $\mathbf{M}^{\prime}$ and is combined with the original laser emission at $1.064 \mu \mathrm{m}$ in the balanced homodyne detector shown in Figs. 2 and 3. The operation of this detector is discussed in detail in Section 4.

\section{DETECTION OF SQUEEZED LIGHT}

To detect the output fluctuations of the field from the OPO cavity, we employ a balanced homodyne detector, ${ }^{35,36}$ which yields directly the quantity $S(\nu, \theta)$. A sketch of the configuration to be considered is shown in Fig. 3 , where the fields are normalized such that $\left\langle\hat{E}^{\dagger} \hat{E}\right\rangle$ represents a photon flux. For a quasi-monochromatic field, such a choice presents no difficulties. ${ }^{37}$ The two photocurrents $i_{1}(t)$ and $i_{2}(t)$ are combined with a phase shift of $180^{\circ}$ in a hybrid junction, and the spectral density $\Phi(\nu)$ of the resulting photocurrent $i(t)$ is recorded, where $\Phi(\nu)$ is given by

$$
\Phi(\nu)=\int\langle\Delta i(t) \Delta i(t+\tau)\rangle e^{-i \nu \tau} \mathrm{d} \tau,
$$

with $\Delta i(t) \equiv i(t)-\langle i\rangle$. Assuming delta function responses at both photodetectors, we find that ${ }^{38}$

$$
\begin{aligned}
\langle\Delta i(t) \Delta i(t+\tau)\rangle= & R_{1} Q_{1}{ }^{2} \delta(\tau)+R_{2} Q_{2}{ }^{2} \delta(\tau)+R_{1}{ }^{2} Q_{1}{ }^{2} \lambda_{11}(\tau) \\
& +R_{2}{ }^{2} Q_{2}{ }^{2} \lambda_{22}(\tau)-R_{1} R_{2} Q_{1} Q_{2} \lambda_{12}(\tau) \\
& -R_{1} R_{2} Q_{1} Q_{2} \lambda_{21}(\tau)
\end{aligned}
$$

where $R_{i}$ is the total counting rate from detector $i=1$ or $2, Q_{i}$ is the total charge per photopulse, $Q_{i}=e g_{i}$, with the $g_{i}$ as the gain function of the detector/amplifier configuration, and $\lambda_{i j}(\tau)$ is one of a set of fourth-order field-correlation functions. In the limit of a strong local-oscillator field $\left\langle\hat{E}_{\mathrm{LO}}{ }^{\dagger}\right.$ $\left.\hat{E}_{\mathrm{LO}}\right\rangle \gg\left\langle\hat{E}_{s}{ }^{\dagger} \hat{E}_{s}\right\rangle$, with $\left\langle\hat{E}_{\mathrm{LO}}\right\rangle=E_{0} e^{i \theta}$, we have $R_{1}=\alpha_{1}{ }^{\prime} R E_{0}{ }^{2}$ and $R_{2}=\alpha_{2}^{\prime} T E_{0}{ }^{2}$, where $\alpha_{i}^{\prime}$ is the detector quantum efficiency and $(R, T)$ are the reflectivity and transmissivity of the beam splitter. The correlations of the field that we wish to investigate are contained within the functions $\lambda_{i j}(\tau)$, which are found from a straightforward application of the theory of photoelectric detection developed by Glauber ${ }^{37-39}$ :

$$
\begin{aligned}
& \lambda_{i j}(\tau)=\frac{\left\langle T: \hat{E}_{i}^{\dagger}(t) \hat{E}_{j}^{\dagger}(t+\tau) \hat{E}_{j}(t+\tau) \hat{E}_{i}(t):\right\rangle}{\left\langle\hat{E}_{i}^{\dagger}(t) \hat{E}_{i}(t)\right\rangle\left\langle\hat{E}_{j}^{\dagger}(t+\tau) \hat{E}_{j}(t+\tau)\right\rangle}, \\
& i, j=1,2 .
\end{aligned}
$$

$\hat{E}_{i}$ refers to the total field incident upon detector $i$ and is obtained by an application of the Fresnel equations for the signal and local-oscillator fields at the beam splitter. Sub- 
stituting the various fields into Eq. (19), assuming the limit of a strong local-oscillator beam, and combining the resultant expressions with Eqs. (17) and (18), we find that the spectral density of photocurrent fluctuations $\Phi$ is related to the spectrum of squeezing $S$ in a remarkably simple fashion, namely,

$$
\Phi(\Omega, \theta)=\left\langle Q_{1} i_{1}+Q_{2} i_{2}\right\rangle\left[1+\rho T_{0} \beta \eta^{2} S(\Omega, \theta)\right],
$$

where the phase $\theta$ is determined by the local-oscillator phase plus a constant offset arising from phase shifts at various optical surfaces. The dimensionless frequency $\Omega=\nu / \Gamma_{1}$ is once again introduced. The factors $\left(\rho, T_{0}, \beta, \eta^{2}\right)$ are all efficiency factors lying in the range $0-1$ and are discussed, in turn, below. For the moment, note that in the case of a signal field in a coherent or vacuum state $S=0$, and we have from Eq. (20) that

$$
\left.\Phi(\Omega, \theta)\right|_{S=0}=\left\langle\left(Q_{1} i_{1}+Q_{2} i_{2}\right)\right\rangle,
$$

where $\left\langle i_{1}\right\rangle=e g_{1} \alpha_{1}{ }^{\prime} R E_{0}{ }^{2}$ and $\left\langle i_{2}\right\rangle=e g_{2} \alpha_{2}{ }^{\prime} T E_{0}{ }^{2}$ are the mean photocurrents in the two channels. Equation (21) implies that, for a vacuum-state input at the signal port, the noise level in the photocurrent $i(t)$ is just the sum of shot-noise levels for the two individual channels. Since this level is readily defined experimentally, we introduce the ratio

$$
\begin{aligned}
R(\Omega, \theta) & \equiv \frac{\Phi(\Omega, \theta)}{\left\langle\left(Q_{1} i_{1}+Q_{2} i_{2}\right)\right\rangle} \\
& =1+\rho T_{0} \beta \eta^{2} S(\Omega, \theta),
\end{aligned}
$$

with $R(\Omega, \theta)=1$ now corresponding to the vacuum noise level.

As for the various efficiency factors, the quantities $\rho$ and $T_{0}$ characterize the escape and propagation of the field from the OPO cavity to the beam splitter. $p$ is given by the ratio of loss through $\mathrm{M}^{\prime}$ to the total cavity loss by all avenues (including $\mathbf{M}^{\prime}$ ) and is equal to the ratio of measured cavity finesse $F$ to the finesse $F_{1}$ inferred from the transmissivity of $\mathrm{M}^{\prime}$. In terms of Eqs. (1), if we take $\Gamma_{1}=\gamma_{1}+\gamma_{1}{ }^{\prime}$ with $\gamma_{1}{ }^{\prime}$ associated with $\mathrm{M}^{\prime}$, then $\rho=\gamma_{1}^{\prime} / \Gamma_{1}$. $T_{0}$, of course, depends on the particular optical elements employed between the OPO cavity and the homodyne detector. For our measurements $\rho$ ranges from $0.70<\rho<0.95$, while $T_{0} \sim 0.94$.

$\beta$ is an efficiency factor that characterizes the balanced homodyne detector and is given by

$$
\beta \equiv R T \frac{\left(\alpha_{1}^{\prime} g_{1}+\alpha_{2}^{\prime} g_{2}\right)^{2}}{\left(R \alpha_{1}^{\prime} g_{1}{ }^{2}+T \alpha_{2}{ }^{\prime} g_{2}{ }^{2}\right)} .
$$

The apparent asymmetry of this expression with respect to the numerator and denominator can be understood in qualitative terms by noting that the numerator arises from the function $\lambda_{i j}(\tau)$ and hence involves the detection of the normally ordered product of two intensities $\left(\sim \alpha^{2} g^{2}\right)$, whereas the denominator is a normalization factor associated with the self-convolution of photoelectric pulses produced by a single-coherent-state intensity in one arm $\left(\sim \alpha g^{2}\right)$. A sum of such terms from each arm then leads to an expression such as given in Eq. (23). Note that in the ideal case we would have $\alpha_{1}^{\prime}=\alpha_{2}^{\prime}=\alpha, g_{1}=g_{2}, R=T=1 / 2$, and hence $\beta=\alpha$, which is just the quantum efficiency of the detectors. For an almost balanced detector with $\alpha_{2}{ }^{\prime}=\alpha_{1}{ }^{\prime}+\delta \alpha, R=1 / 2+\epsilon$, $T=1 / 2-\epsilon$, and $g_{2}=g_{1}+\delta g$, we find to lowest order in $\delta \alpha / \alpha$, $\epsilon$, and $\delta g / g$ that $\beta=\left(\alpha_{1}{ }^{\prime}+\alpha_{2}{ }^{\prime}\right) / 2 \equiv \bar{\alpha}$, independent of gain and reflectivity variations. For the InGaAs photodiodes employed in our experiment, $\alpha_{1}{ }^{\prime}=0.91 \pm 0.02$ and $\alpha_{2}{ }^{\prime}=0.87 \pm$ 0.02 . The deviation $\epsilon$ is less than 0.02 .

The factor $\eta^{2}$ in Eq. (22) expresses the homodyne efficiency at the detectors and is given by the mode overlap across the assumed uniform surface of the photodiode ${ }^{40}$ :

$$
\eta=\left|\iint \mathrm{d}^{2} \mathbf{r} U^{*}(\mathbf{r}) V(\mathbf{r})\right|,
$$

where $\hat{E}_{\mathrm{LO}}(\mathbf{r}, t)=\hat{E}_{\mathrm{LO}}(t) U(\mathbf{r})$ and $\hat{E}_{s}(\mathbf{r}, t)=\hat{E}_{s}(t) V(\mathbf{r}) . \quad \eta^{2}$ enters Eq. (22) rather than $\eta$ since we are dealing with photocurrent fluctuations that derive from correlation functions that are fourth order in the field amplitude. The phase of the mode integral in Eq. (24) is absorbed into the localoscillator phase $\theta$ as a constant offset. [In writing Eqs. (18)(19), the explicit integrals over the detector surface were omitted for the sake of brevity.] To measure the heterodyne efficiency, we employ an auxiliary beam at $\omega$ that is approximately mode matched to the OPO cavity and that is injected through $\mathrm{M}$ and transmitted through $\mathrm{M}^{\prime}$. The spatial filtering provided by the cavity in transmitting only the $\mathrm{TEM}_{00}$ component of this injected beam helps ensure that the localoscillator beam is matched to the actual $\mathrm{TEM}_{00}$ mode of the cavity. The injected beam can be frequency offset from the laser emission at $\omega$ with an acousto-optic modulator. We can thus determine $\eta$ either by a homodyne measurement of the visibility of the fringe produced from the interference of local oscillator and cavity transmission or, in the case of a frequency offset of the injected beam, by a heterodyne measurement that compares the rf beat to the shot-noise level set by the local oscillator. The two techniques agree for the value of $\eta$ to within $\pm 5 \%$. Our usual technique for determining $\eta$ is by using the rf-beat technique, which requires an absolute knowledge of the power entering the signal port (the local-oscillator power is common to both the measurement of the shot-noise level and of the amplitude of the coherent $\mathrm{rf}$ beat). Denoting $D$ as the ratio of $\mathrm{rms}$ beat current at the offset frequency $f$ to the rms level of shot-noise current at $f$ (obtained by blocking the signal port), we find that

$$
\eta^{2}=\frac{D^{2} \hbar \omega}{\alpha P_{s}} \Delta f
$$

where $P_{s} \sim\left\langle\hat{E}_{s}^{\dagger} \hat{E}_{s}\right\rangle$ is the power delivered from the cavity to the photodetector, $\alpha$ is the quantum efficiency of the photodiode, $\Delta f$ is the detection bandwidth, and $\omega$ the frequency of the field at $1.06 \mu \mathrm{m}$. For all our measurements $\eta \approx 0.95$.

Although the above analysis assumes a coherent state for the local oscillator, unfortunately real lasers often produce a field with fluctuations far above this level. These excess local-oscillator fluctuations can be incorporated into the preceding analysis by introducing the function

$$
S_{\mathrm{LO}}(\nu) \equiv \int \mathrm{d} \tau\left\langle T: \hat{Y}(t), \hat{Y}(t+\tau):>e^{-i \nu \tau},\right.
$$

with $\hat{Y}=\hat{E}_{\mathrm{LO}}+\hat{E}_{\mathrm{LO}}{ }^{\dagger}$. For fluctuations of the local-oscillator field that are small compared with the mean value of the field, we find an additional contribution to $R(\nu, \theta)$ given by 


$$
\frac{\left(\alpha_{1}{ }^{\prime} g_{1} R-\alpha_{2}{ }^{\prime} g_{2} T\right)^{2}}{\alpha_{1}{ }^{\prime} g_{1}{ }^{2} R+\alpha_{2}{ }^{\prime} g_{2}{ }^{2} T} S_{\mathrm{LO}}(\nu) \equiv \delta_{-} S_{\mathrm{LO}}(\nu),
$$

which expresses an increase in the noise level (above the vacuum level) associated with the amplitude noise of the local oscillator. These excess fluctuations are, however, greatly suppressed by the balanced homodyne arrangement as can be seen by considering the case $\alpha_{2}{ }^{\prime}=\alpha_{1}{ }^{\prime}+\delta \alpha, g_{2}=g_{1}+$ $\delta g$, and $R=1 / 2+\epsilon, T=1 / 2-\epsilon$, for which

$$
\delta_{-} \approx \alpha_{1}{ }^{\prime}\left(2 \epsilon-\frac{\delta \alpha}{2 \alpha_{1}{ }^{\prime}}-\frac{\delta g}{2 g_{1}}\right)^{2} .
$$

In the ideal case, the detector is perfectly balanced and $\epsilon=$ $\delta \alpha=\delta g=0$. However, as Eq. (27) demonstrates, excess local-oscillator noise is suppressed with reasonable vigor even for nonideal balancing. In our measurements, $\delta_{-}<$ $10^{-3}$.

Considerations of excess local-oscillator noise are extremely important because the observation of noise levels below the vacuum noise level $[R(\nu, \theta)<1]$ is only as reliable as the accuracy to which the vacuum level can itself be fixed. One common practice is to employ a variety of sources as the local oscillator (helium-neon laser, incandescent lamp, ...), which one has some reason to believe should be quiet in their noise spectrum above some particular radio frequency $\nu_{0}$. If for a range of $\nu>\nu_{0}$ one obtains the same noise levels for all sources, including the actual local oscillator to be employed in the squeezing measurements, the conjecture is that this noise level is in fact the vacuum or shot-noise level. While this procedure is certainly a valuable one, it is, nonetheless, fraught with uncertainties associated with the spectral content of the various sources and with the focusing geometry on the photodiodes. (Collection efficiency and effective capacitance depend on the physical locations within the photodiode at which photocarriers are generated and hence on the spectral content and geometry of the illumination.) It is often the case that a discrepancy between sources is noted for the noise level observed for equal dc photocurrents. Once this discrepancy is eliminated by one of a variety of fixes (altered focusing geometry, spectral filtering of incandescent source, ...), success is declared without any totally convincing argument that the inferred noise level is, in fact, the vacuum noise level. Since it is extremely difficult to calibrate accurately the absolute shot-noise level for a given source, we advocate a new procedure for determining the vacuum noise level that seems to be free of most of the ambiguities described above.

Our technique is straightforward and employs a summing junction $\sum_{+}$in conjunction with the differencing junction $\sum$ - indicated in Fig. 3. We alternately measure the spectral densities (for a vacuum state at the signal port of the balanced detector)

$$
\chi_{+}(\nu)=\left(Q_{1}\left\langle i_{1}\right\rangle+Q_{2}\left\langle i_{2}\right\rangle\right)\left[1+\delta_{+} S_{\mathrm{LO}}(\nu)\right]
$$

and

$$
\chi_{-}(\nu)=\left(Q_{1}\left\langle i_{1}\right\rangle+Q_{2}\left\langle i_{2}\right\rangle\right)\left[1+\delta_{-} S_{\mathrm{LO}}(\nu)\right],
$$

with

$$
\delta_{ \pm}=\frac{\left(\alpha_{1}{ }^{\prime} g_{1} R \pm \alpha_{2}{ }^{\prime} g_{2} T\right)^{2}}{\left(\alpha_{1}{ }^{\prime} g_{1}{ }^{2} R+\alpha_{2}{ }^{\prime} g_{2}{ }^{2} T\right)}=\alpha(R \pm T)^{2},
$$

where the second equality follows for $g_{1}=g_{2}, \alpha_{1}{ }^{\prime}=\alpha_{2}{ }^{\prime}=\alpha$, and $R+T=1 . \quad \chi_{ \pm}(\nu)$ is obtained by combining $i_{1}$ and $i_{2}$ first with $0^{\circ}$ phase shift $\left(\chi_{+}\right)$and second with $180^{\circ}$ phase shift $\left(\chi_{-}\right)$, with the attenuation of the hybrid junctions separately calibrated at the frequency of interest. For $\alpha_{1}{ }^{\prime} \sim \alpha_{2}{ }^{\prime} \sim 1$ and $\delta_{-} \ll 1$, the extent to which $\chi_{+}$and $\chi_{-}$coincide is the extent to which one can infer $S_{\mathrm{LO}}=0$. The ratio $\delta_{-} / \delta_{+}$can be determined from a measurement in which a coherent amplitude modulation $\left(\gg S_{\mathrm{LO}}\right)$ is imposed on the local-oscillator beam at the frequency $\nu$ of interest; this ratio together with the ratio $\chi_{+} / \chi_{-}$leads to a quantitative expression for $S_{\mathrm{LO}}$,

$$
S_{\mathrm{LO}}=\frac{\chi_{+} / \chi_{-}-1}{\delta_{+}\left(1-\frac{\chi_{+} \delta_{-}}{\chi_{-} \delta_{+}}\right)} \approx\left(\chi_{+} / \chi_{-}-1\right) / \alpha,
$$

where the last step follows for efficient detection and small excess fluctuations. We stress that this procedure permits an in situ determination of $S_{\mathrm{LO}}$ for the actual local-oscillator geometry employed in the squeezing measurements.

For the Nd:YAG laser and balanced homodyne-detector arrangement shown in Fig. 3, the ratio $\delta_{+} / \delta_{-}>10^{3}$ for frequencies around $1.6 \mathrm{MHz}$, at which most of our measurements of squeezing are made, while $\chi_{+} / \chi_{-}=1 \pm 0.01$. Hence $\left|S_{\mathrm{LO}}\right|<0.011$ for $\alpha=0.90$. Furthermore, the contribution of excess amplitude noise is suppressed by greater than $30 \mathrm{~dB}$ by the balanced homodyne detector with the $180^{\circ}$ phase shift that is actually employed in the experiment so that $\delta_{-}\left|S_{\mathrm{LO}}\right| \sim 10^{-5}$. Fine balancing of the gains $\left(g_{1}, g_{2}\right)$ is accomplished with a wideband variable attenuator in one arm.

As a further confirmation that the observed noise level for a vacuum-state input to the signal port is indeed the vacuum noise level and not a level set by excess fluctuations on the local-oscillator beam, we made measurements with a spectrally filtered incandescent lamp (10-nm bandpass). We examined both the noise levels in the individual arms and the noise levels produced by two independent lamp arrangements (one in each arm) in the balanced configuration. For a laser focused to underfill the detector area $(80-\mu \mathrm{m}$ diameter), the photocurrent noise associated with the laser illumination is approximately $1.2 \mathrm{~dB}$ higher than that produced by the spectrally filtered source for identical dc photocurrents. However, if the local-oscillator field is defocused such that the detector area is overfilled and the illumination geometry is more nearly equivalent to that of the lamp, the noise levels of lamp and laser coincide to within $\pm 5 \%$. We stress that the issue here is not one of detector saturation but is rather that photocarriers generated in the edge regions of the photodiode can contribute to the dc photocurrent but not to the high-frequency noise spectrum. Hence the overfilled photodiode appears quieter than the underfilled one for the same dc photocurrents.

We conclude this section by collecting together the important parameters that enter Eq. (22). For the various cavity and detection configurations that we employ, the efficiency factors are as follows: $0.70<\rho<0.95, T_{0} \sim 0.94, \beta \sim \alpha \sim$ $0.89, \eta^{2} \sim 0.90$. In addition, we note that, at the rf frequencies of interest in our measurements, the excess fluctuations of the local oscillator are negligible. 


\section{OBSERVATION OF SQUEEZED STATES}

As indicated in Figs. 2 and 3, our observation of squeezing centers on an analysis of the spectral distribution of fluctuations in the signal formed by the subtraction of the two photocurrents $\left(i_{1}, i_{2}\right)$ produced by the photodiodes $\left(D_{1}, D_{2}\right)$. Because of its central importance to the discussion that follows, we rewrite Eq. (22) as

$$
R(\Omega, \theta)=1+\rho T_{0} \beta \eta^{2} S(\Omega, \theta),
$$

where the phase $\theta$ is varied by scanning a mirror in the localoscillator path that is mounted on a piezoelectric ceramic. Each of the efficiency factors $\left(\rho, T_{0}, \beta, \eta^{2}\right)$ was discussed in turn in Section 4. $S(\Omega, \theta)$ is the spectrum of squeezing of the signal beam $\hat{E}_{s}$ emitted by the OPO. Our objectives are twofold in the analysis that follows: (1) we wish to observe noise reductions below the vacuum level that are as large as possible, and (2) for a given noise level we wish to infer $S$ as accurately as possible from the measured values of $R$ and the set $\left(\rho, T_{0}, \beta, \eta^{2}\right)$.

Examples of the phase dependence of the rms noise voltage $V(\theta)$ from the balanced homodyne receiver as a function of local-oscillator phase $\theta$ at fixed analysis frequency $\Omega$ are shown in Figs. 4-6. For each of the figures, the dashed line at the level 1.0 corresponds to the noise voltage $V_{0}$ set by vacuum fluctuations and is obtained either by blocking the signal input to the balanced detector or by tuning the temperature of the lithium niobate crystal to shift the cavity resonance at $\omega_{1}$ from coincidence with the locked resonance at $\omega_{2}$. (Note that the injected beam discussed in Sections 3 and 4 is blocked for all our measurements of squeezing described here.) Values of $V(\theta)$ below the dashed lines in Figs. 4-6 represent observations of squeezing of the incident sig-

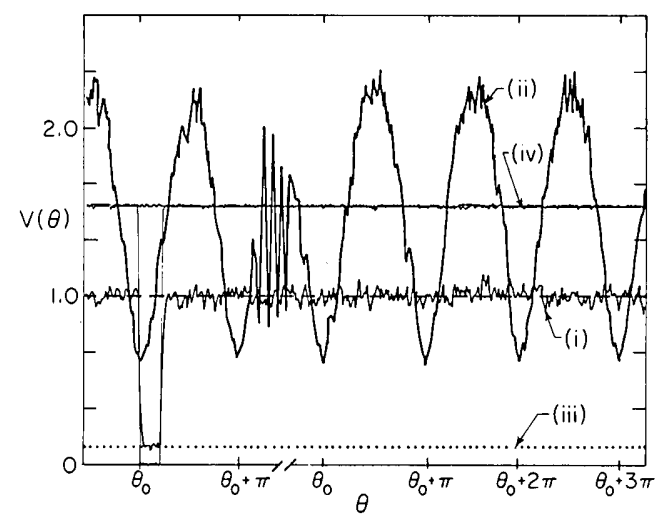

Fig. 4. Dependence of $\mathrm{rms}$ noise voltage $V(\theta)$ on local-oscillator phase $\theta$ for the signal from the balanced homodyne detector shown in Fig. 3. With the output of the OPO blocked, the vacuum field entering the detector produces the noise voltage $V_{0}$ labeled by (i) with no sensitivity on 0 . With the OPO input present, trace (ii) exhibits phase-sensitive deviations both below and above the vacuum level, with the dips below trace (i) representing a $61 \%$ reduction in noise power relative to the vacuum level. Trace (iii) is the amplifier noise level. Note that for traces (i)-(iii) the ordinate is linear in noise voltage (amplitude). Trace (iv) is actually two curves almost superimposed that give the levels of dc photocurrent (with zero at the bottom of the figure) during the acquisition of traces (i) and (ii). For traces (i)-(iii), $\nu / 2 \pi=1.6 \mathrm{MHz}$. The sharp feature is generated by the flyback of the piezoelectric ceramic used to scan the local-oscillator phase. The time for the entire sweep is $0.2 \mathrm{sec}$, the analysis bandwidth is $100 \mathrm{kHz}$, and time constants for the postdetection video filters are $1.5 \times 10^{-4}$ and $5.0 \times 10^{-4} \mathrm{sec}$, respectively.

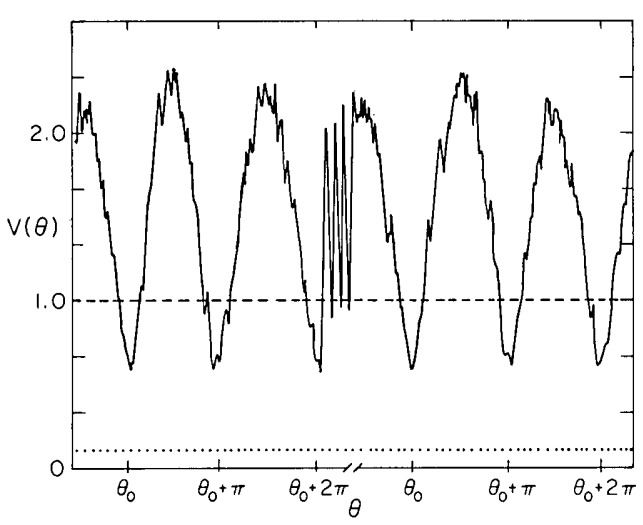

Fig. 5. Dependence of noise voltage $V(\theta)$ on local-oscillator phase $\theta$ for the signal beam produced by the subthreshold OPO. Operating conditions are as in Fig. 4, with traces (i) and (iv) deleted; the dashed line is the vacuum level obtained by multiple averaging.

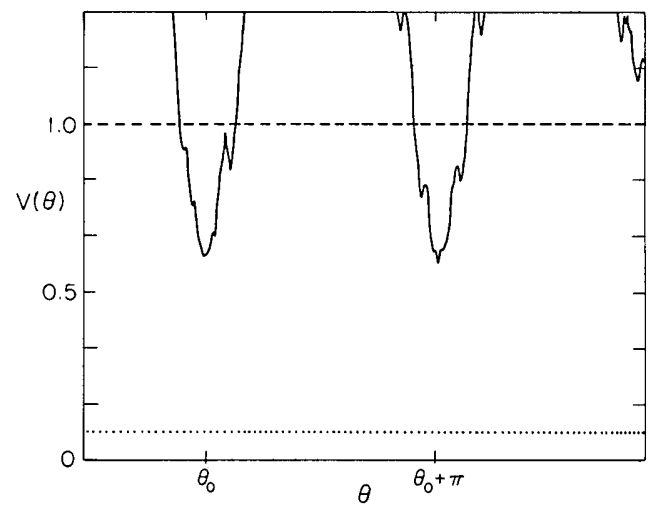

Fig. 6. Dependence of noise voltage $V(\theta)$ on local-oscillator phase $\theta$ for the signal beam produced by the subthreshold OPO. The linear scale in rms noise voltage is expanded relative to Figs. 4 and 5 to display the deviation below the vacuum level (dashed line) more clearly. Reductions in noise power of $63 \%$ relative to the vacuum level are shown. $\nu / 2 \pi=1.6 \mathrm{MHz}$. Analysis bandwidth and video filter time constants are as in Fig. 4.

nal field. In Fig. 4 we explicitly show all the traces that are stored in a given measurement of squeezing. The dotted line (iii) near the bottom of the figure corresponds to the amplifier noise level that at $1.6 \mathrm{MHz}$ is a level $-20 \mathrm{~dB}$ below the vacuum noise level $V_{0}$. Curve (ii) exhibiting the large phase-dependent variations is, of course, the result $V(\theta)$ obtained with the squeezed input present at the signal port. The phase-independent trace (i) fluctuating about the level 1.0 is the vacuum noise level for a single sweep that was initiated by blocking the local-oscillator beam briefly near $\theta_{0}$ on the left-hand side of the figure. The lightly drawn level (iv) between 1.0 and 2.0 that is almost constant is actually a signal proportional to the dc photocurrent and is measured directly from the detector in one arm of the homodyne receiver, with zero current at the bottom of the figure. Two traces are very nearly superimposed-one recorded simultaneously with the sweep that generated $V(\theta)$ and one simultaneous with the sweep that generated $V_{0}$. These two nearly overlapping traces demonstrate that, although the dc photocurrent and the local-oscillator power are essentially constant, the associated spectral densities of photocurrent fluctuations are greatly different for squeezed light and for the vacuum field, as shown by $V(\theta)$ and $V_{0}$. Although the actual traces of the vacuum level and of the total photocurrent are 
not shown in Figs. 5 and 6, for the sake of clarity, they have in fact been recorded precisely, as above. (We also looked for squeezing with a $0^{\circ}$ summing junction in place of the $180^{\circ}$ difference junction. In this case the phase-sensitive variations shown in Figs. 4-6 should be suppressed by roughly the factor $\delta_{-}$. As expected in the $0^{\circ}$ case, no squeezing was observed).

Assuming that the variances associated with the thermal noise of the amplifier $V_{A}$ and with that of the photocurrent add as variances for independent random processes, we find that the ratio $R(\Omega, \theta)$ can be obtained directly from our data as

$$
R(\theta)=\frac{V^{2}(\theta)-V_{A}^{2}}{V_{0}^{2}-V_{A}^{2}},
$$

where fixed $\Omega$ is assumed and we recall that the degree of squeezing is expressed in terms of noise power not noise voltage. In fact, the contribution from $V_{A}$ is negligible for most of our measurements. We collect a large number of traces in 62 trace sequences, with typical traces shown in Figs. 4-6 and from these data construct histograms of minimum and maximum noise levels. From the mean and variances of these histograms, we quote values of $R_{ \pm}$and associated uncertainties, where $R_{-}=R\left(\theta_{-}\right)$and $R_{+}=R\left(\theta_{+}\right)$denote the values of the minimum and maximum noise level. For the data obtained under the operating conditions of Figs. 4 and 5 , we find $R_{-}=0.39 \pm 0.03$ and $R_{+}=4.8 \pm 0.4$. From Fig. 6, we find $R_{-}=0.37 \pm 0.03$. For the data of Fig. 6, $\rho=$ $0.85 \pm 0.05, T_{0}=0.94 \pm 0.02, \beta=0.89 \pm 0.03$, and $\eta^{2}=0.95 \pm$ 0.05 , so that from Eq. (32) $S_{-}=-(0.93 \pm 0.08)$. These data have been quite reproducible over the past several months. The minimum noise level $R_{-}$is consistently around $R_{-}=$ 0.37 , corresponding to $S_{-} \sim-0.90$.

In order to investigate the dependence of $S_{-}=S_{-}\left(\Omega, \theta_{-}\right)$ on the pump ratio $r=P_{2} / P_{0}=d^{2}$, with $P_{0}$ the incident power at $0.53 \mu \mathrm{m}$ required to reach the threshold for parametric oscillation, we have recorded data as shown in Figs. 4-6 over a range of incident pump powers $P_{2}$. The result of our measurements is shown in Fig. 7, where the solid curve is the theoretical prediction from Eq. (8), evaluated at the mea-

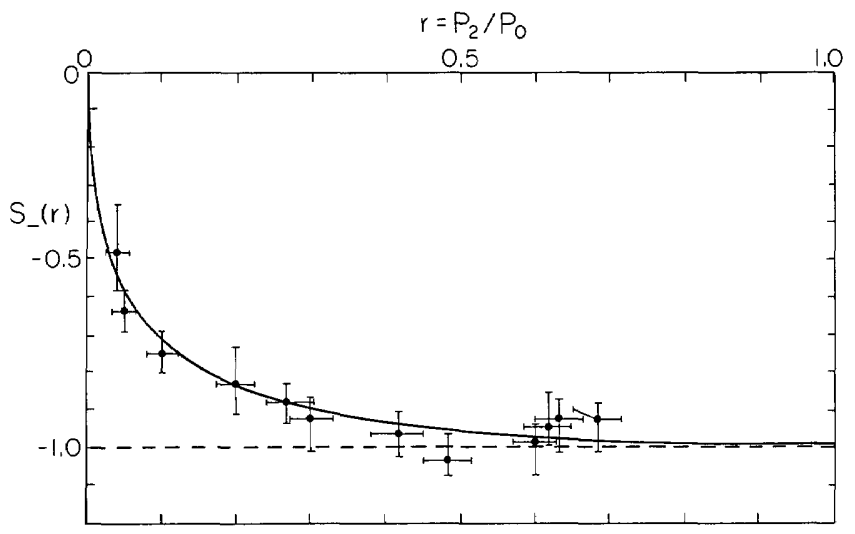

Fig. 7. Comparisons of the values $S_{-}(r)$ derived from the measurements with those given by theory (solid curve) for fixed $\Omega=0.21$ with the measured values $\rho T_{0} \beta \eta^{2}=0.52$ and $P_{0}=30 \mathrm{~mW}$. Perfect squeezing occurs for $S_{-}(\Omega)=-1$ and is indicated by the dashed line. The uncertainties shown for each point derive from uncertainties in the determination of $R_{-}$and of $P_{2}$ at a given operating point and do not include the overall uncertainties in $\rho, T_{0}, \beta, \eta^{2}$, or $P_{0}$. surement offset frequency $\Omega=0.21(\nu / 2 \pi=1.2 \mathrm{MHz})$. For the data, $\rho=0.70 \pm 0.07, T_{0}=0.93 \pm 0.02, \beta=0.89 \pm 0.03, \eta^{2}$ $=0.90 \pm 0.10$, and $P_{0}=(30 \pm 5) \mathrm{mW}$. Given these values, $r$ is then determined from the measured input power $P_{2}$ and

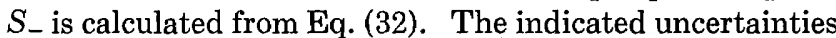
derive from uncertainties in the measurement of $R_{-}$and $P_{2}$ at a given operating point and do not include the overall uncertainties in $\left(\rho, T_{0}, \beta, \eta^{2}\right)$ or $P_{0}$. We stress that the comparison of theory and experiment shown in Fig. 7 is absolute with no adjustable parameters and that no fitting was attempted within the allowed uncertainties. From Fig. 7 , we conclude that, in the absence of the avoidable linear losses expressed by $\left(\rho, T_{0}, \beta, \eta^{2}\right)$, the field would in fact be squeezed by more than tenfold for operation with $r \sim 0.5$. Stated somewhat differently, the intracavity field of our optical parametric oscillation as it now exists is such that more than tenfold squeezing would be produced in the output field if the intracavity field were to decay through only a single output coupler. For the losses that do exist in the current OPO cavity, we infer a degree of squeezing for the actual field emitted through $\mathrm{M}^{\prime}$ of $S_{-} e=\rho S_{-} \sim-0.8$, corresponding to fivefold squeezing.

We have also explored the dependence of $S_{-}(\Omega) \equiv S_{-}(\Omega$, $\theta_{-}$) on $\Omega$. For a fixed value of $\Gamma=0.55$ and with the measured values of $\left(\rho, T_{0}, \beta, \eta^{2}\right)$, we have confirmed that this frequency dependence is in reasonable agreement with the dependence predicted by Eq. (8). In particular, the measured width in frequency $\Gamma_{0}$ over which the squeezing persists is clearly increased from the value $\Gamma_{1}$ associated with the cavity in the absence of nonlinear interaction to a value more nearly that given by Eq. (8), $\Gamma_{0}=\Gamma_{1}\left(1+\sqrt{P_{2} / P_{0}}\right)$.

Although until this point we have concentrated on the quantities $\left(R_{-}, S_{-}\right)$that express the squeezing of the field in terms of reduction of fluctuations, we can also examine the enhancement in fluctuations in the conjugate field quadrature expressed by $\left(R_{+}, S_{+}\right)$. Theoretically, the field emitted by the OPO is predicted to be in a minimum-uncertainty state in the sense that ${ }^{18,19,28}$

$$
\left[1+S_{+}(\Omega)\right]\left[1+S_{-}(\Omega)\right]=1 \text {. }
$$

More generally, the commutation relation associated with the quadrature-phase amplitudes of the field $\hat{E}_{s}(t)$ implies the uncertainty relation ${ }^{29}$

$$
\left[1+S_{+}(\Omega)\right]\left[1+S_{-}(\Omega)\right] \geq 1 \text {. }
$$

Thus a squeezed state need not be a minimum uncertainty state. Those systems for which equality is achieved in expression (34) represent the best squeezing allowed by quantum mechanics. Since for a single-mode field the class of minimum uncertainty states are unitarily equivalent to the vacuum state, ${ }^{8-10}$ we describe the output field of the OPO as a squeezed-vacuum state.

Figure 8 is a plot constructed from our measurements to explore the question of minimum uncertainty for the field of the OPO. As before, the quantities $S_{ \pm}$are inferred in absolute terms and without adjustment from the measured values of $R_{ \pm}$and $\left(\rho, T_{0}, \beta, \eta^{2}\right)$. The operating conditions are the same as those described in conjunction with Fig. 7. The solid curve in Fig. 8 is the hyperbola defined by Eq. (33). The agreement between theory and experiment is quite remarkable. Since Eq. (33) follows as a direct consequence of 


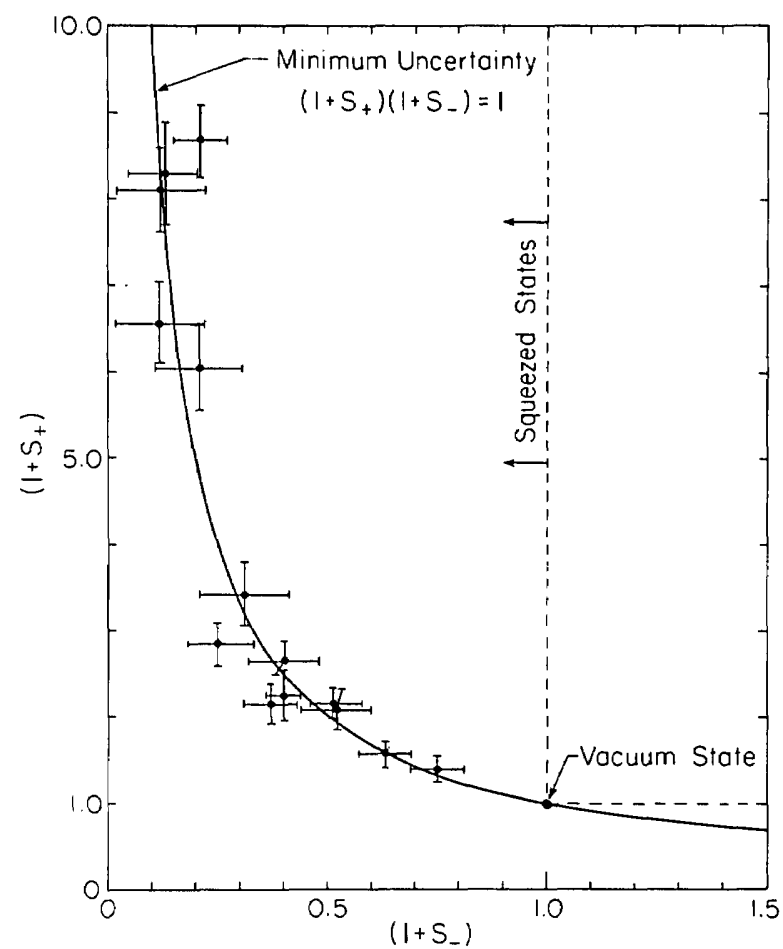

Fig. 8. Variances $\left(1+S_{+}\right)$and $\left(1+S_{-}\right)$determined from measurements such as those shown in Figs. 4-6, as discussed in the text. The solid curve is the hyperbola $\left(1+S_{+}\right)\left(1+S_{-}\right)=1$, which defines a class of minimum-uncertainty states. Squeezed states are those states for which $\left(1+S_{ \pm}\right)<1$ and that lie in the region bounded by the hyperbola and the dashed lines.

the nonzero commutator of the quadrature-phase amplitudes, ${ }^{29}$ we suggest that Fig. 8 represents not only a rather striking demonstration of the Heisenberg uncertainty principle for light but also is about as close as one can come to the direct measurement of the commutation relation itself.

In addition to our observations of squeezed-state generation with intracavity lithium niobate, we have also conducted a preliminary search for squeezing with an intracavity crystal of barium sodium niobate. Under conditions similar to those of Figs. 4-6, noise reductions of approximately $35 \%$ were obtained. This work was hampered by a number of problems, chief among them being the biaxial nature of the crystal. However, even this modest noise reduction relative to that achieved with lithium niobate should serve to indicate that squeezed-state generation by parametric downconversion is not restricted to the particular details of our experiments with $\mathrm{MgO}: \mathrm{LiNbO}_{3}$.

\section{CONCLUSION}

We have described experiments that apply the process of parametric downconversion in a subharmonic OPO to reduce noise levels in homodyne detection by more than $60 \%$ $(-4 \mathrm{~dB})$ relative to the vacuum noise level. The spectrum of squeezing $S(\Omega, \theta)$ extracted from our measurements [Eq. (32) and Fig. 7] indicates that the observed noise reductions resulted from a field that would be squeezed more than tenfold in the case of lossless propagation and detection ( $\rho=$ $\left.T_{0}=\beta=\eta=1\right)$. The uncertainty product $\left(1+S_{+}\right)\left(1+S_{-}\right)$ derived from the measurements and plotted in Fig. 8 suggests that the field state produced in the lossless circum- stance would be a minimum-uncertainty state (a squeezedvacuum state). The absolute, quantitative comparisons with theory that we have presented support the contention that the process of parametric downconversion in the subthreshold OPO generates a squeezed state in a fashion adequately modeled by a simple parametric Hamiltonian, free from competing noise processes encountered in other squeezed-state experiments. Degradation of the squeezing seems to occur by way of a variety of straightforward linear attenuation mechanisms, each of which can be largely eliminated given sufficient technical resources. However, even for the current cavity losses $(\rho=0.85)$, we emphasize that the field emerging from the OPO is squeezed by approximately fivefold for pumping levels $r \geq 0.5$. This is a degree of squeezing that can be employed in certain experiments (e.g., atomic spectroscopy) in spite of our lack of ability to detect it efficiently.

Beyond the demonstration of the feasibility of achieving a large degree of squeezing, our work sets the stage for a number of exciting applications in optical physics. Indeed, by making use of the squeezed light of the OPO we recently achieved signal detection with an improvement of $3.0 \mathrm{~dB}$ in sensitivity beyond the vacuum-state or shot-noise limit. ${ }^{41}$

\section{ACKNOWLEDGMENTS}

This research was supported by the Venture Research Unit of British Petroleum, International, and by the U.S. Office of Naval Research. We gratefully acknowledge the contributions of J. L. Hall, Kun-Chi Peng, and Huifa Wu to this research program. The $\mathrm{MgO}: \mathrm{LiNbO}_{3}$ crystal was generously provided by Crystal Technology and the $\mathrm{Ba}_{2} \mathrm{NaNb}_{5} \mathrm{O}_{15}$ crystal by the Shanghai Institute of Ceramics. The dualband coatings were skillfully fabricated by Virgo Optics.

\section{REFERENCES}

1. H. Takahasi, Adv. Commun. Syst. 1, 227 (1965).

2. W. H. Louisell, A. Yariv, and A. E. Siegman, Phys. Rev. 124, 1646 (1961).

3. B. R. Mollow and R. J. Glauber, Phys. Rev. 160, 1076 (1967).

4. R. Graham, Springer Tracts in Modern Physics (Springer-Verlag, Berlin, 1973), Vol. 66.

5. D. C. Burnham and D. L. Weinberg, Phys. Rev. Lett. 25, 84 (1970).

6. B. R. Mollow, Phys. Rev. A 8, 2684 (1973).

7. S. Friberg, C. K. Hong, and L. Mandel, Phys. Rev. Lett. 54, 2011 (1985).

8. D. Stoler, Phys. Rev. D 1, 3217 (1970); 4, 1925 (1971).

9. E. Y. C. Lu, Lett. Nuovo Cimento 2, 1241 (1971).

10. H. P. Yuen, Phys. Rev. A 13, 2226 (1976).

11. C. M. Caves, Phys. Rev. D 23, 1693 (1981).

12. D. F. Walls, Nature 306, 141 (1983).

13. M. M. Fejer, G. A. Magel, and R. L. Byer, Appl. Opt. 24, 2362 (1985); Y. S. Luh, R. S. Feigelson, M. M. Fejer, and R. L. Byer, J. Cryst. Growth 78, 135 (1986).

14. B. Yurke, P. Grangier, R. E. Slusher, and M. J. Potasek, Phys, Rev. A 35, 3586 (1987).

15. R. G. Smith, in Lasers: A Series of Advances, A. K. Levine and A. DeMaria, eds. (Dekker and Marcel, New York, 1976), Vol. 4.

16. R. L. Byer, in Quantum Electronics: A Treatise, H. Rabin and C. L. Tang, eds. (Academic, New York, 1975), Vol. 1, Part B, p. 587.

17. B. Yurke, Phys. Rev. A 29, 408 (1984).

18. M. J. Collett and C. W. Gardiner, Phys. Rev. A 30, 1386 (1984); 31, 3761 (1985).

19. C. W. Gardiner and C. M. Savage, Opt. Commun. 50, 173 (1984). 
20. L.-A. Wu, H. J. Kimble, J. L. Hall, and H. Wu, Phys. Rev. Lett. 57,2520 (1986).

21. R. E. Slusher, L. W. Hollberg, B. Yurke, J. C. Mertz, and J. F. Valley, Phys. Rev. Lett. 55, 2409 (1985).

22. R. M. Shelby, M. D. Levenson, S. H. Perlmutter, R. G. DeVoe, and D. F. Walls, Phys. Rev. Lett. 57, 691 (1986); B. L. Schumaker, S. H. Perlmutter, R. M. Shelby, and M. D. Levenson, Phys. Rev. Lett. 58, 357 (1987).

23. M. W. Maeda, P. Kumar, and J. H. Shapiro, Opt. Lett. 12, 161 (1987).

24. S. Machida, Y. Yamamoto, and Y. Itaya, Phys. Rev. Lett. 58, 1000 (1987).

25. M. G Raizen, L. A. Orozco, Min Xiao, T. L. Boyd, and H. J. Kimble, Phys. Rev. Lett. 59, 198 (1987).

26. P. D. Drummond, K. J. McNeil, and D. F. Walls, Opt. Acta 27, 321 (1980); 28, 211 (1981).

27. M. D. Levenson, R. M. Shelby, and S. H. Perlmutter, Opt. Lett. 10,514 (1985).

28. M. J. Collett and D. F. Walls, Phys. Rev. A 32, 2887 (1985).

29. C. M. Caves and B. L. Schumaker, Phys. Rev. A 31, 3068 (1985); 31, 3093 (1985); in Quantum Optics IV, J. H. Harvey and D. F. Walls, eds. (Springer-Verlag, New York, 1986), p. 20.
30. F. Zernike and J. E. Midwinter, Applied Nonlinear Optics (Wiley, New York, 1973).

31. G. D. Boyd and D. A. Kleinman, J. Appl. Phys. 39, 3597 (1968).

32. L.-A. Wu and H. J. Kimble, J. Opt. Soc. Am. B 2, 697 (1985).

33. K.-C. Peng, L.-A. Wu, and H. J. Kimble, Appl. Opt. 24, 938 (1985).

34. R. W. P. Drever, J. L. Hall, F. V. Kowalski, J. Hough, G. M. Ford, A. J. Munley, and H. Ward, Appl. Phys. B 31, 97 (1983).

35. H. P. Yuen and V. W. S. Chan, Opt. Lett. 8, 177 (1983); B. L. Schumaker, Opt. Lett. 9, 189 (1984).

36. J. H. Shapiro, IEEE J. Quantum Electron. 21, 237 (1985).

37. H. J. Kimble and L. Mandel, Phys. Rev. A 30, 844 (1984).

38. L. Mandel, in Optics in Four Dimension-1980, M. A. Machado and L. M. Narducci, eds., AIP Conf. Proc. No. 65 (American Institute of Physics, New York, 1981).

39. R. J. Glauber, in Quantum Optics and Electronics, C. deWitt, A. Blandim, and C. Cohen-Tannoudji, eds. (Gordon \& Breach, New York, 1965).

40. L. Mandel and E. Wolf, J. Opt. Soc. Am. 65, 413 (1975).

41. M. Xiao, L.-A. Wu, and H. J. Kimble, Phys. Rev. Lett. 41, 278 (1987).

\section{H. J. Kimble}

H. J. Kimble is an associate professor of physics at the University of Texas at Austin. He received the B.S. degree in physics from Abilene Christian University in 1971 and the Ph.D. degree in physics from the University of Rochester in 1978. From 1977 to 1979 he was at General Motors Research Laboratories as an associate senior research scientist. His research is generally centered in the area of experimental quantum optics. 